\title{
Controle de Botryosphaeria ribis causador de seca de ponteiro em Corymbia citriodora, com extratos vegetais e fungicidas
}

\author{
Adimara Bentivoglio Colturato ${ }^{1}$; Edson Luiz Furtado ${ }^{1 *}$
}

Departamento de Produção Vegetal/ Defesa Fitossanitária, Faculdade de Ciências Agronômicas, Unesp/ Botucatu. Caixa Postal 235. ${ }^{1}$ CNPq; *Bolsista CNPq Autor para correspondência: Adimara B. Colturato (adimara@gmail.com) Data de chegada: 18/12/2010. Aceito para publicação em: 07/06/2011.

\section{RESUMO}

Colturato, A.B.; Furtado, E.L. Controle de Botryosphaeria ribis causador de seca de ponteiro em Corymbia citriodora, com extratos vegetais e fungicidas Summa Phytopathologica, v.37, n.3, p.137-141, 2011.

A seca de ponteiros é uma doença que vem acarretando danos severos em plantas de eucalipto, causando cancros ao longo do ramo principal. O objetivo deste trabalho foi avaliar o efeito de fungicidas e extratos vegetais no controle de Botryosphaeria ribis. O teste "in vitro" dos fungicidas foi inteiramente casualizado com oito tratamentos: carbendazim, clorotalonil, difenoconazole, picoxystrobin + ciproconazole, ciproconazole, azoxystrobin, picoxystrobin e testemunha, quatro doses: $1 \mu \mathrm{g} / \mathrm{mL}, 10 \mu \mathrm{g} / \mathrm{mL}, 100$ $\mu \mathrm{g} / \mathrm{mL}$ e $1000 \mu \mathrm{g} / \mathrm{mL}$; com cinco repetições. Após homogeneização do meio foram vertidas para as placas, repicado um disco de meio de cultura contendo o patógeno para estas placas e mantidas em BOD a $25^{\circ} \mathrm{C}$ por cinco dias. A avaliação foi feita através de medição diária do crescimento radial do micélio em centímetros. O delineamento experimental do controle químico a campo foi em fatorial $5 \times 3$, com cinco fungicidas e três métodos de aplicação (poda, pincelado e pulverizado), com quatro repetições. Foram feitas quatro aplicações, com intervalo de quinze dias. Os tratamentos foram: 1. azoxystrobin, 2. carbendazim, 3. clorotalonil + tiofanato-metílico, 4. difenoconazole e 5. testemunha. Simultaneamente foram feitas avaliações seguindo uma escala de notas de 1 a 6 . O delineamento experimental do teste "in vitro" dos extratos vegetais foi em fatorial $5 \times 4$, com três repetições. Os tratamentos avaliados foram os extratos de: mil folhas, melão de são caetano, eucalipto, álcool e testemunha, nas concentrações de $5,10,15$ e $20 \%$. Os extratos e o álcool foram misturados ao meio de cultura previamente autoclavado, sendo estes colocados em placas de Petri. A avaliação foi feita através de medição diária do crescimento radial do micélio em centímetros. No teste com mudas o delineamento experimental foi em fatorial $2 \times 2 \times 4$, sendo utilizado no experimento de duas procedências, dois modos de tratamento (preventivo e convencional) e quatro produtos para o controle (extrato de melão de são caetano, extrato de Corymbia citriodora, álcool e água). A inoculação do patógeno foi feita no caule das mudas. Foram feitas aplicações dos produtos e avaliações semanais. A avaliação foi feita através da contagem de plantas doentes. O ingrediente ativo carbendazin foi o que mostrou os melhores resultados "in vitro" diferindo estatísticamente dos outros tratamentos pelo teste Tukey a $1 \%$ de probabilidade; seguido pelo clorotalonil e difenoconazole. Todos os ingredientes ativos avaliados mostraramse superiores a testemunha. A campo, azoxystrobin foi superior aos demais tratamentos e não houve diferença significativa entre os métodos de aplicação. "In vitro", os extratos de mil folhas, melão e eucalipto não diferiram entre si pelo teste de Tukey a $5 \%$ de probabilidade. O álcool proporcionou a maior inibição do crescimento micelial e diferiu estatisticamente dos outros tratamentos utilizados. As concentrações de 10,15 e $20 \%$ dos extratos não diferiram entre si, mas foram superiores a concentração de $5 \%$. No teste em mudas, a aplicação preventiva mostrou-se superior a aplicação curativa, sendo que o álcool e o extrato de C. citriodora não diferiram entre si, mas foram superiores ao extrato de melão-de-são-caetano. Todos os produtos foram superiores a testemunha.

Palavras-chave adicionais: Corymbia citriodora, Dothyorella sp., controle químico e controle com extratos vegetais.

\begin{abstract}
Colturato, A.B.; Furtado, E.L. Control of Botryosphaeria ribis the causative agent of tip drougth using plant extracts and fungicides. Summa Phytopathologica, v.37, n.3, p.137-141, 2011.

Tip drought is a disease that has been causing severe damages to eucalyptus trees, resulting in canker along the branches. The aim of the present study was to evaluate the effect of fungicides and plant extracts on the control of Botryosphaeria ribis. The in vitro test of fungicides was completely randomized with eight treatments: carbendazim, chlorothalonil, difenoconazole, picoxystrobin + ciproconazol, ciproconazol, azoxystrobin, picoxystrobin and control, four doses: $1 \mu \mathrm{g} / \mathrm{mL}, 10 \mu \mathrm{g} / \mathrm{mL}, 100 \mu \mathrm{g} / \mathrm{mL}$ and $1000 \mu \mathrm{g} / \mathrm{mL}$, with five replicates. After homogenization of the medium, they were plateds, recultured in a disk with culture medium containing the pathogen and

kept in BOD at $25^{\circ} \mathrm{C}$ for five days. The evaluation was done by daily measuring the mycelium radial growth in centimeters. The experimental design of chemical control in the field was in $5 \times 3$ factorial arrangement, five fungicides and three application methods, with four replicates. Four applications were done with fifteen-day intervals. Treatments were: 1. azoxystrobin, 2. carbendazim, 3 . chlorothalonil + thiophanate-methyl, 4. difenoconazole and 5 . control. Simultaneously evaluations were done following a scale from 1 to 6 , conform the intensity of symptoms. The experimental design in vitro using plant extracts was in $5 \times 4$, factorial arrangement, with
\end{abstract}


three replicates. The evaluated treatments were extracts from: a thousand leaves sao caetano melon, eucalyptus, alcohol and control, at the concentrations of 5, 10, 15 and $20 \%$. The extracts and the alcohol were mixed with previously autoclaved culture medium, which was then plated in Petri dishes. The evaluation was done by daily measuring the mycelium radial growth in centimeters. In the test with seedlings the experimental design was in $2 \times 2 \times 4$, factorial arrangement, and two cultivars were used besides two treatment modes (preventive and curative) and four control products (são caetano melon extract, $C$. citriodora extract, alcohol and water). The pathogen was inoculated into the stem of seedlings. Applications and evaluations were done weekly. The evaluation was done by counting the diseased plants. The active ingredient carbendazin showed the best results in vitro statistically differing from the other treatments according to Tukey's test at $1 \%$ probability, followed by chlorothalonil and difenoconazole. All active ingredients were superior to the controls. In the field, azoxystrobin was superior to the remaining treatments and there was no significant difference between application methods. In vitro the extracts from thousand leaves, melon and eucalyptus did not differ according to Tukey's test at 5\% probability. Alcohol led to the greatest mycelial growth inhibition and differed significantly from the other treatments. The concentrations 10,15 and $20 \%$ extracts did not differ but were superior to the concentration of $5 \%$. In the test with seedlings, the preventive application was superior to healing application, while alcohol and $C$. citriodora extract did not differ, but were superior to the sao caetano melon extract. All products were superior to control.

Keywords: Corymbia citriodora, Dothyorella sp., chemical control, control using plant extracts.

A seca de ponteiro e os cancros formados por Botryosphaeria ribis têm ganhado importância devido aos danos causados. Uma vez instalada a doença o seu controle torna-se difícil.

Em manga, B. ribis causa severa quebra do ponteiro em $83 \%$ das plantas inoculadas (10). Em pistache, foram avaliados diversos fungicidas, porém, estes apresentaram apenas controle marginal. O fungicida iprodione mostrou-se efetivo no controle de Botryosphaeria em pistache (13). A perda de eficácia de iprodione devido à resistência tem sido relatada para diversos patógenos como Botrytis cinerea, Alternaria alternata e Sclerotinia minor após vários anos de uso intensivo (4, 6, 12). Zhonghua Ma et al. (2001), estudando a resistência de $B$. dothidea de pistache à iprodione constataram que o patógeno desenvolveu resistência em meio de cultura, indicando que o fungo tem uma propensão de contornar a ação do fungicida. Aplicações múltiplas dos fungicidas azoxystrobin e tebuconazole promoveram um controle eficaz para pistache, mas o rápido desenvolvimento de resistência a fungicidas em $B$. dothidea preocupa os produtores de pistache da Califórnia $(11,12)$.

Experimentos conduzidos em coqueiro visando determinar o efeito de fungicidas sobre a incidência de Lasiodiplodia theobromae, constouse uma variação da incidência da doença entre $2 \%$ a $33 \%$ em coqueiros pulverizados com a mistura de benomyl $-0,1 \%$ i.a e carbendazim $0,1 \%$ i.a. (9).

Segundo Leung citado por Zhonghua Ma et al.(16), o uso de cultivares resistentes talvez seja o método mais durável para o controle de B. dothidea.

Com o avanço do sistema de produção, a busca por novas práticas agrícolas que substituam os métodos convencionais de controle de doenças aumentou, buscando métodos alternativos aos fungicidas tradicionais, que sejam eficientes com o mínimo de impacto ambiental e danos à saúde dos seres humanos (6).

Os extratos vegetais de plantas medicinais têm apresentado resultados satisfatórios quanto à ação antifúngica. Diferentes concentrações do extrato etanólico de espinheira-santa inibiu em mais de $10 \%$ o crescimento micelial de Fusarium oxysporum, nas três concentrações avaliadas $(0,2 ; 0,4$ e $0,6 \mathrm{mg} / \mathrm{mL})$ e estimulou em $30 \%$ o crescimento micelial de Colletotrichum acutatum na concentração de $0,2 \mathrm{mg} / \mathrm{mL}$ (3).

Cunico et al. (5), avaliando o potencial antifúngico de Ottonia martiana demonstrou que o extrato desta planta inibiu em mais de $40 \%$ o crescimento de Cylindrocladium spathulatum em folhas de erva-mate. $\mathrm{O}$ extrato bruto de $C$. citriodora a partir da concentração de $20 \%$ proporcionou a inibição do crescimento micelial de Phytophthora sp., Colletotrichum sublineolum, Sclerotium rolfsii, Rhizoctonia solani e Alternaria alternata (3). O mesmo autor em 2004 constatou que a partir da concentração de $5 \%$ o extrato aquoso autoclavado de $C$. citriodora inibiu em mais de $90 \%$ a germinação de esporos, enquanto para o extrato não autoclavado a inibição máxima foi de $75 \%$. Tendo em vista os poucos dados sobre o controle de seca de ponteiro o presente trabalho teve por objetivo avaliar controle químico e o controle com o uso de extratos vegetais.

\section{MATERIAL E MÉTODOS}

Os testes "in vitro" e o teste com mudas foram realizados no Departamento de Produção Vegetal da Faculdade de Ciências Agronômicas, UNESP de Botucatu/SP. O experimento de campo foi realizado na Fazenda Juliana, em Botucatu - SP.

\section{Teste "in vitro" com extratos vegetais}

$\mathrm{Na}$ obtenção do extrato alcoólico, folhas frescas das plantas foram submetidas à secagem em estufa a $180^{\circ} \mathrm{C}$ por 1 hora. Em seguida foram trituradas e misturadas a uma solução de álcool a 96\%, deixadas no escuro por 10 dias e filtrada em gaze. A partir da solução dos extratos estes foram misturados ao meio de cultura previamente autoclavado, colocados em placas de Petri e após o endurecimento do meio foi colocado um disco do fungo e as placas mantidas em BOD a $26^{\circ} \mathrm{C}$ por quatro dias. As concentrações de 5, 10, 15 e $20 \%$ foram avaliadas. O delineamento experimental foi fatorial $5 \times 4$, com três repetições. Os tratamentos avaliados foram os extratos de mil folhas (Achillea millefolium L.), melão de são caetano (Momordica charantia L.), eucalipto (Corymbia citriodora), álcool e testemunha. A avaliação foi feita através de medição diária do crescimento radial do micélio em centímetros.

\section{Teste em mudas de eucalipto}

Foram utilizadas mudas de Corymbia citriodora de duas procedências, sementes originadas de Minas Gerais e do Estado de São Paulo (IPEF). O delineamento experimental foi em fatorial $2 \times 2 \times$ 4, sendo utilizado no experimento de duas procedências (São Paulo e Minas Gerais), dois modos de tratamento (preventivo e convencional) e quatro produtos para o controle (extrato de melão de são caetano, extrato de Corymbia citriodora, álcool e água). Foi utilizada a 
concentração de $10 \%$ para aplicação dos produtos. Para a inoculação foi feito um pequeno ferimento no caule das mudas e em seguida colocado um disco de $8 \mathrm{~mm}$ do patógeno e envolto por algodão umedecido em água destilada fixado por filme plástico de pvc. Após uma semana da inoculação, os algodões foram retirados. Foram feitas aplicações dos produtos e três avaliações, com intervalo de sete dias. A avaliação foi feita através da contagem de plantas doentes, ou seja, plantas com os sintomas da seca de ponteiro. Os dados foram transformados em $(x+a ́)^{1 / 2}$ e analisados pelo programa Estat.

\section{Teste "in vitro" com fungicidas}

Para a realização do teste foram preparadas soluções estoques em água destilada e esterilizada e diluídas conforme o necessário para a obtenção das concentrações desejadas $(1 \mu \mathrm{g} / \mathrm{mL}, 10 \mu \mathrm{g} / \mathrm{mL}, 100 \mu \mathrm{g} /$ $\mathrm{mL}$ e $1000 \mu \mathrm{g} / \mathrm{mL}$ ). Sendo utilizado os fungicidas: carbendazim, clorotalonil, difenoconazole, picoxystrobin + ciproconazole, ciproconazole, azoxystrobin, picoxystrobin e testemunha. Alíquotas das soluções foram adicionadas ao meio de cultura fundente $\left(45^{\circ}\right.$ $50^{\circ} \mathrm{C}$ ), homogeneizou-se, verteu em placas de Petri, esperou-se o meio solidificar e após 24 horas foram transferidos para as placas um disco de aproximadamente $5 \mathrm{~mm}$ de diâmetro do patógeno. As placas foram mantidas em BOD a $25^{\circ} \mathrm{C}$ por cinco dias. As avaliações foram feitas através da medição diária do crescimento radial do micélio em centímetros, até que o crescimento micelial do patógeno cobrisse toda a superfície do meio de cultura, no interior da placa.

\section{Teste a campo com fungicidas}

O delineamento experimental foi em fatorial $5 \times 3$, com cinco tratamentos e três modos de aplicação, com quatro repetições. Os tratamentos consistiram dos seguintes princípios ativos: 1. azoxystrobin, 2. carbendazim, 3. clorotalonil + tiofanato-metílico, 4. difenoconazole e 5. testemunha (água). As dosagens utilizadas foram as recomendadas pelo fabricante. Foram feitas quatro aplicações, em intervalos de 15 dias.

Foram feitas oito avaliações seguindo uma escala de notas, como se segue:

0. plantas sem seca de ponteiros, ou seja, com dominância apical;

1. plantas com ramos laterais afetados;

2. plantas com lesões atingindo ramo principal, mas sem anelamento;

3. plantas com lesões atingindo ramo principal, mas com anelamento;

4. plantas exibindo mais de um anelamento no ramo principal;

5. planta sem o ápice da planta.

6. seca do ponteiro ou planta morta devido a seca do ponteiro

Com os valores de incidência, calculou-se a área abaixo da curva.

Depois efetuado o cálculo da percentagem de controle relativo e em seguida feito análise estatística utilizando-se do programa Estat, através do teste de médias Tukey. Para análise, os dados foram transformados em arc sen $\sqrt{x / 100}$. Os experimentos repetidos duas vezes.

\section{RESULTADOS E DISCUSSÃO}

\section{Teste com extratos vegetais}

No teste in vitro, os extratos de mil folhas, melão e eucalipto não diferiram entre si pelo teste de Tukey a $5 \%$ de probabilidade (Tab. 01). O álcool proporcionou a maior inibição do crescimento micelial e diferiu estatisticamente dos outros tratamentos utilizados. As concentrações de 10, 15 e $20 \%$ dos extratos não diferiram entre si, mas foram superiores a concentração de $5 \%$. O álcool a partir da concentração de $10 \%$ inibiu completamente o crescimento micelial do patógeno, e os extratos de mil folhas, $C$. citriodora e melão-de-sãocaetano inibiram o crescimento micelial a partir da concentração de $15 \%$. Bonaldo et al. (3), trabalhando com diferentes concentrações do extrato bruto de E. citriodora sobre os fungos $R$. solani, S. rolfsii, $C$. sublineolum, Phytophthora sp. e A. alternata, também observaram inibição do crescimento micelial a partir de $20 \%$ da concentração.

$\mathrm{Na}$ avaliação com mudas de Corymbia notou-se na primeira avaliação que não houve diferença significativa entre a procedência das mudas utilizadas (Tab. 02). Aplicando-se os produtos preventivamente antes da inoculação do patógeno, a incidência foi menor do que aplicando de forma curativa. Não houve diferença significativa pelo Teste de Tukey a $5 \%$ de probabilidade entre os produtos utilizados, mas todos diferiram estatisticamente da testemunha. $\mathrm{O}$ mesmo pode ser observado na segunda avaliação, com a diferença de que na segunda avaliação o extrato de melão-de-sãocaetano não diferiu da testemunha.

$\mathrm{Na}$ terceira avaliação, todas as plantas tratadas apresentavam-se doentes, iniciando com a seca do ponteiro e progredindo para a seca total da muda. Isso demonstrou que os extratos utilizados não conseguiram controlar o desenvolvimento do patógeno.

\section{Teste com fungicidas}

Todos os ingredientes ativos foram superiores a testemunha. Carbendazin apresentou os melhores resultados inibindo o crescimento micelial do patógeno a partir da concentração de 10 $\mu \mathrm{g} / \mathrm{mL}$ (Tabela 03), seguido por clorotalonil e difenoconazole, mostrando-se um ingrediente potencial no controle da seca de ponteiro. Em Lasiodiplodia theobromae em manga, foi constatado que os ingredientes ativos carbendazim e tiofanato metílico a 1 $\mathrm{mg} /$ $\mathrm{mL}$ proporcionaram inibição do crescimento micelial do patógeno (7). Em abricó japonês, o tiofanato metílico inibiu o crescimento micelial e a germinação de conídios e controlou o desenvolvimento

Tabela 1. Avaliação in vitro do controle de Botryosphaeria ribis com extratos vegetais.

\begin{tabular}{|c|c|c|c|c|c|}
\hline \multirow[b]{2}{*}{ Tratamentos } & \multirow[b]{2}{*}{$5 \%$} & \multirow[b]{2}{*}{$10 \%$} & \multicolumn{2}{|l|}{ Doses } & \multirow[b]{2}{*}{ Média* } \\
\hline & & & $15 \%$ & $20 \%$ & \\
\hline Álcool & 1,13 a $\mathrm{C}$ & 1,14 a $\mathrm{B}$ & 1,14 a $\mathrm{B}$ & 1,14 a $\mathrm{B}$ & $1,13 \mathrm{C}$ \\
\hline C. citriodora & 2,84 a $\mathrm{A}$ & $1,31 \mathrm{~b} \mathrm{~B}$ & 1,14 b B & 1,14 b $\mathrm{B}$ & $1,60 \mathrm{~B}$ \\
\hline Mil Folhas & 2,73 a $\mathrm{A}$ & 1,42 b B & $1,14 \mathrm{~b} \mathrm{~B}$ & 1,14 b B & $1,61 \quad \mathrm{~B}$ \\
\hline
\end{tabular}

* Médias seguidas da mesma letra não diferem entre si pelo Teste de Tukey a 5\% de probabilidade. Letras minúsculas diferem entre si na linha e letras maiúsculas na coluna. 
Tabela 2. Avaliação do controle de Botryosphaeria ribis em mudas de eucalipto com extratos vegetais.

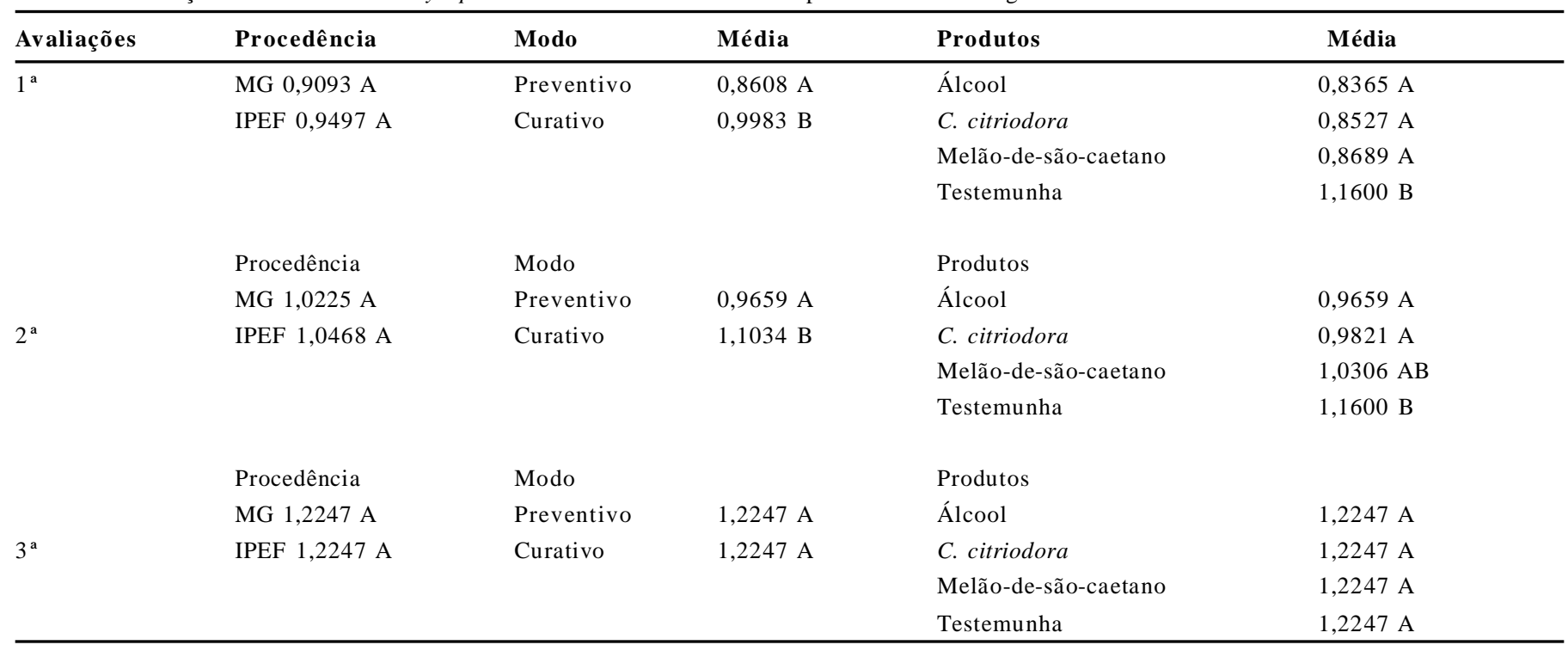

* Médias seguidas da mesma letra não diferem entre si pelo Teste de Tukey a 5\% de probabilidade. Letras maiúsculas diferem entre si na oluna.

Tabela 3. Avaliação in vitro do controle de Botryosphaeria ribis com fungicidas.

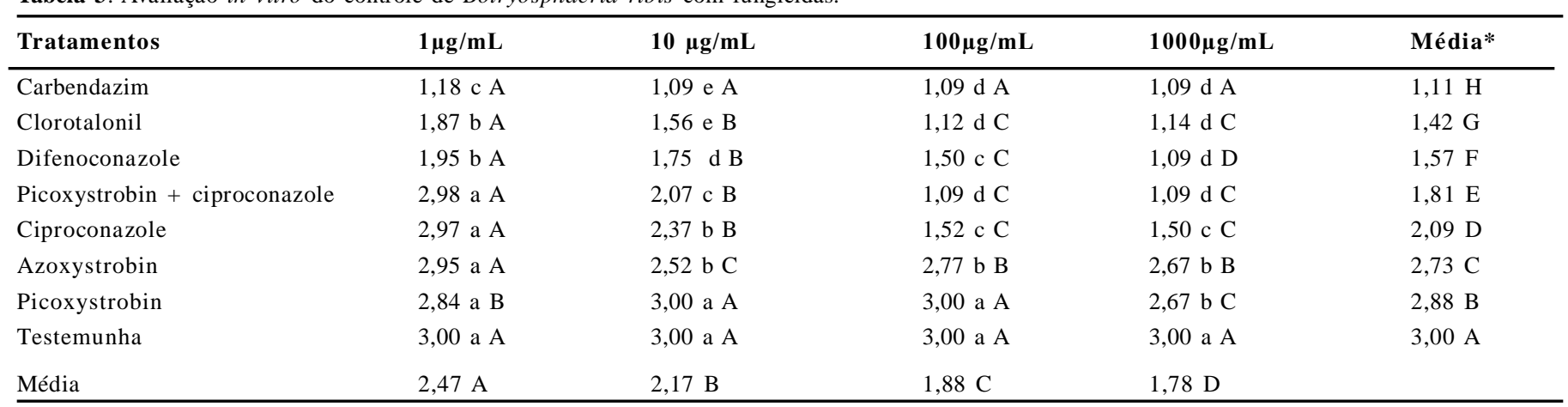

* Médias seguidas da mesma letra não diferem entre si pelo Teste de Tukey a 5\% de probabilidade. Letras minúsculas diferem entre si na coluna e letras maiúsculas na linha.

Tabela 4. Porcentagem de controle relativo médio de Botryosphaeria ribis com fungicidas, em relação ao tratamento Testemunha.

\begin{tabular}{|c|c|c|c|c|c|c|c|}
\hline \multicolumn{8}{|c|}{ Avaliações } \\
\hline Tratamentos & $2^{\mathrm{a}}$ & $3^{a}$ & $4^{\mathrm{a}}$ & $5^{a}$ & $6^{a}$ & $7^{\mathrm{a}}$ & $\mathbf{8}^{\mathbf{a}}$ \\
\hline Carbendazin & $84,05 \mathrm{~A}$ & 81,88 AB & $84,41 \mathrm{~A}$ & $85,08 \mathrm{~A}$ & $83,76 \mathrm{~A}$ & $88,35 \mathrm{~A}$ & $84,42 \mathrm{~A}$ \\
\hline Difenoconazole & $88,35 \mathrm{~A}$ & $85,83 \mathrm{~A}$ & $82,54 \mathrm{~A}$ & $86,59 \mathrm{~A}$ & $83,20 \mathrm{~A}$ & $76,42 \mathrm{~A}$ & $82,60 \mathrm{~A}$ \\
\hline Clorotalonil & $88,35 \mathrm{~A}$ & $90,00 \mathrm{~A}$ & $90,00 \mathrm{~A}$ & $88,35 \mathrm{~A}$ & $90,00 \mathrm{~A}$ & $82,64 \mathrm{~A}$ & $77,13 \mathrm{~A}$ \\
\hline Azoxystrobin & $78,80 \mathrm{~A}$ & $69,60 \quad \mathrm{~B}$ & 66,89 В & 69,92 B & $67,11 \quad \mathrm{~B}$ & $70,26 \mathrm{~A}$ & $71,61 \mathrm{~A}$ \\
\hline \multicolumn{8}{|c|}{ Avaliações } \\
\hline & $2^{\mathrm{a}}$ & $3^{\mathbf{a}}$ & $4^{a}$ & $5^{a}$ & $6^{a}$ & $7^{\mathbf{a}}$ & $8^{\mathbf{a}}$ \\
\hline Poda & $87,53 \mathrm{~A}$ & $85,84 \mathrm{~A}$ & $84,75 \mathrm{~A}$ & $84,95 \mathrm{~A}$ & $82,42 \mathrm{AB}$ & $77,82 \mathrm{~A}$ & $78,92 \mathrm{~A}$ \\
\hline Pulverizado & $79,94 \mathrm{~A}$ & $74,35 \quad \mathrm{~B}$ & $73,98 \quad$ B & $77,37 \mathrm{~A}$ & $73,45 \quad \mathrm{~B}$ & $75,24 \mathrm{~A}$ & $77,82 \mathrm{~A}$ \\
\hline Pincelado & $87,19 \mathrm{~A}$ & $85,30 \mathrm{~A}$ & $84,15 \mathrm{~A}$ & $84,95 \mathrm{~A}$ & $87,19 \mathrm{~A}$ & $85,19 \mathrm{~A}$ & $80,07 \mathrm{~A}$ \\
\hline
\end{tabular}

* Dados transformados em arc sen $\sqrt{x / 100}$.

de Botryosphaeria dothidea e L. theobromae (8).

Os ingredientes ativos carbendazin, clorotalonil, difenoconazole e azoxystrobin quando empregados a campo demonstraram visualmente uma redução na severidade da doença (Tab. 04). Após a $1^{\mathrm{a}}$ aplicação dos produtos (momento da $2^{\text {a }}$ avaliação), pode-se notar que azoxystrobin apresentou melhor controle da doença, porém, não diferiu estatisticamente de carbendazim. Nas $4^{\mathrm{a}}, 5^{\mathrm{a}}$ e $6^{\mathrm{a}}$ avaliações, azoxystrobin foi superior aos demais tratamentos, sendo que os 
demais tratamentos não diferiram entre si. Nas $7^{\mathrm{a}}$ e $8^{\mathrm{a}}$ avaliações não houve diferença significativa entre os tratamentos, demonstrando que talvez fossem necessárias mais aplicações dos produtos, pois o patógeno continuou a se desenvolver. O método de aplicação pulverizado, após as $1^{\mathrm{a}}$ e $2^{\mathrm{a}}$ aplicações, demonstrou ser mais eficiente que os demais (Tab.04). Porém após a $4^{\mathrm{a}}$ aplicação ( $5^{\mathrm{a}}$ avaliação) não houve diferença significativa entre os modos de aplicação.

Em pistache, múltiplas aplicações dos fungicidas azoxystrobin e tebuconazole promoveram um controle eficiente de B. dothidea (14, 15). Em maçã, $B$. dothidea é controlada principalmente devido à eficácia de captan e de fungicidas benzimidazóis (1). Em manga, carbendazim foi mais efetivo do que o tiofanato metílico e phosetyl-Al na redução da infecção de L. theobromae, suprimindo a exsudação da goma, dieback e murcha, resultando ainda um aumento significativo no crescimento vegetativo das plantas (7). Por causa da sua natureza destrutiva, Botryosphaeria tem demonstrado ser de difícil controle em plantas de eucalipto.

Conclui-se que azoxystrobin e carbendazim apresentaram os melhores resultados no controle de Botryosphaeria ribis; sendo sugeridos para o manejo desta doença. Os extratos vegetais não foram eficientes no controle da doença em mudas.

\section{REFERÊNCIA BIBLIOGRÁFICAS}

1.Biggs, A.R, Effect of inoculum concentration and calcium salts on infection of apple fruit by Botryosphaeria dothidea. Plant disease, St. Paul, v.88, n.2,p.147-151, 2004.

2.Bonaldo, S.M.; Schwan-Estrada, K.R.F.; Stangarlin, J.R.; Tessmann, D.J.; Scapim, C.A. Fungitoxicidade, atividade ellicitora de fitoalexinas e proteção de pepino contra Colletotrichum lagenarium, pelo extrato aquoso de Eucaliptus citriodora. Fitopatologia Brasileira, Brasília-DF, v.29, n.2, p.128-134, 2004.

3.Bonaldo, S.M.; Schwan-Estrada, K.R.F.; Stangarlin, J.R.; Cruz, M.E.S.; Fiori-tutida, A.C.G. Contribuição ao estudo das atividades antifúngicas e elicitora de fitoalexinas em sorgo e soja por eucalipto (Eucalyptus citriodora). Summa Phytopatologica, Botucatu, v.33, n.4, p.383-387, 2007.

4.Chastagner, G.A.; Vassey, W.E. Occurrence of iprodione-tolerant Fusarium nivale under field conditions. Plant Disease, St. Paul, v. 66, p. $112-114,1982$.

5.Cunico, M.M.; Miguel, O.G.; Miguel, M.D.; Carvalho, J.L.S.; Peitz, C.; Auer, C.G.; Grigoletti Júnior, A. Estudo da atividade antifúnfica de Ottonia martiana Miq., Piperaceae: um teste in vivo. Visão Acadêmica, Curitiba, v. 4, n.2, p.77-82, 2003.

6.Detweiler, A.R.; Vargas, J.M.; Danneberger, T.K. Resistance of Sclerotinia homoeocarpa to iprodione and benomyl. Plant Disease, St. Paul, v.67, p. 627-630, 1983.

7.Khanzada, M.A.; Lodhi, A.M.; Shahzad, S. Chemical control of Lasiodiplodia theobromae, the causal agent of mango decline in sindh. Pakistan Journal of Botany, Karachi, v. 37, n. 4, p. 1023-1030, 2005.

8.Li, Hong-Ye; Cao, Rou-Bin; Mu, Yong-Tan. In vitro inhibition of Botryosphaeria dothidea and Lasiodiplodia theobromae, and chemical control of gummosis disease of Japanese apricot and peach trees in Zhejiang Province, China. Disponível em: $<$ http:/ /www.sciencedirect.com/science > Acesso em: 15 jan 2009.

9.Ram, C. Efeito de fungicidas aplicados em mistura sobre incidência da queima-das folhas e produção do coqueiro. Fitopatologia Brasileira, Brasília-DF, v. 18, p. 264, 1993.

10.Ramos, L.J.; Davenport, T.L.; McMillan, R.T.; Lara, S.P. The resistance of mango (Mangifera indica) cultivars to tip dieback disease in Florida. Plant Disease, St Paul, v.81, n.5, p.509$514,1997$.

11.Schwann-Estrada, K.R.F. Uso de plantas medicinais no controle de doenças de plantas. Fitopatologia Brasileira, Brasília-DF, v.28, suplemento, p.54-56, 2003.

12.Solel, Z.; Timmer, L.W.; Kimchi, M. Iprodione resistance of $A l$ ternaria alternata pv. Citri from Minneola tangelo in Israel and Florida. Plant Disease, St. Paul, v.80, p. 291-293, 1996.

13.Zhonghua Ma, Luo Yong, Michailides, T.J. Resistance of Botryosphaeria dothidea to iprodione. Plant Disease, St. Paul, v. 85, n. 2, p. 183-188, 2001.

14.Zhonghua Ma, Morgan, D.P.; Michailides, T.J. Effects of water stress on Botryosphaeria blight of pistachio caused by Botryos pheria dothidea. Plant Disease, St. Paul, v. 85, n.7, p.745-749, 2001 .

15.Zhonghua Ma, Michailides, T.J. Characterization of Botryosphaeria dothidea isolates collected from pistachio and other plant hosts in California. Phytopathology, St. Paul, v.92, n.5, p.519526, 2002 .

16.Zhonghua Ma, Luo Yong, Michailides, T.J. Spatiotemporal changes in the population structure of Botryosphaeria dothidea from California pistachio orchards. Phytopathology, St. Paul, v.94, n. 4, p. 326-332, 2004. 